\title{
Moral Threshold Model is Universal? Initial Evidence from China's Collectivist Culture
}

\author{
Weilong Xiao ${ }^{1,2, *}$ \\ Binghai Sun $\mathbb{D}^{1, *}$ \\ Hui Zhou ${ }^{3}$ \\ Liting Fan ${ }^{1,2}$ \\ Changkang Sun' \\ Yanhong Shao ${ }^{4}$ \\ 'College of Teacher Education, Zhejiang \\ Normal University, Jinhua, Zhejiang, \\ People's Republic of China; ${ }^{2}$ Key \\ Laboratory of Intelligent Education \\ Technology and Application of Zhejiang \\ Province, Zhejiang Normal University, \\ Jinhua, Zhejiang, People's Republic of \\ China; ${ }^{3}$ Jinhua Advanced Research \\ Institute, Jinhua, Zhejiang, People's \\ Republic of China; ${ }^{4}$ Zhejiang International \\ Studies University, Hangzhou, Zhejiang, \\ People's Republic of China \\ * These authors contributed equally to \\ this work
}

Purpose: People choose actions that maintain their moral self-regard. This paper explains how one's moral actions influence moral self-regard. The moral threshold model (MTM) has been proposed by scholars and tested using a limited sample. However, whether the MTM is universally applicable among people is still unknown.

Participants and Methods: Our sample comprised 1761 individuals living in China, a collectivist culture, who were recruited to test the two main hypotheses of the MTM. Participants engaged in a hypothetical investment task.

Results: 1) When the beneficiary was a prosocial cause, participants showed stronger preferences for smaller guaranteed positive payouts over larger uncertain ones; 2) as compared to making decisions for charities, when a participant made decisions exclusively for themselves, the maximum potential benefit was more likely to influence participant behavior. Conclusion: The current study provides initial evidence for the validity of using the worst outcome avoidance (WOA) hypothesis of MTM among members of China's collectivist culture, indicating that the WOA hypothesis of MTM may have universal application.

Keywords: collectivist culture, morality, moral self-regard, prosocial behavior, egoistic behavior

\section{Introduction}

Moral self-regard describes how people evaluate their own moral merits or the extent to which they believe they are moral. ${ }^{1}$ People try to create a consistent moral identity. $^{2,3}$ In other words, they spare no effort in proving they are morally good, thus preserving moral self-regard. They approve of prosocial behaviors, ${ }^{4,5}$ rejecting dishonesty, ${ }^{2}$ protecting their moral integrity, ${ }^{6}$ and holding those who upstage them by doing something nobler in low regard. ${ }^{7}$ All of these combine to serve one purpose: to make others and themselves believe they are moral.

Although moral behaviors are indeed fascinating, they come into conflict with people's desires (ie, money). Therefore, it is understandable that people will not always strive to be perfectly moral. Instead, they will settle for "moral enough." ing that achieving the status of "moral enough" will not harm their moral identity. ${ }^{9-12}$ Additionally, people have claim to have good reasons for choosing the most moral course of action $^{13,14}$ and to have strong justifications for their immoral actions. ${ }^{1,15}$

Given that people do not have a strict internal moral code, they still perceive themselves as moral actors even when they act in immoral ways. However, they cannot go too far in the opposite direction. In other words, people's behavior must meet a minimum internal moral threshold. Otherwise, their moral self-regard will
Correspondence: Changkang Sun College of Teacher Education, Zhejiang inhua, 321004 , Zhejiang, People's Email II58067980@qq.com

Yanhong Shao

Zhejiang International Studies University, 299 Liuhe Road, Xihu District, Hangzhou, 310013, Zhejiang Province, People's Republic of China

Email yhshao2003@I26.com 
be damaged. This leads to the question: What is the threshold? To be more specific, how much morality should people exhibit to be sufficiently moral?

\section{The Moral Threshold Model (MTM)}

To address the question above, Zlatev et al ${ }^{16}$ proposed the moral threshold model (MTM). The model establishes essential requirements for people when making prosocial decisions. Typically, these requirements can ensure people's moral self-regard. The MTM depends on two underlying hypotheses.

The first MTM hypothesis is the worst outcome avoidance (WOA) hypothesis. It states that in the process of making prosocial decisions that enhance moral self-regard, people strive to ensure that the consequence of their choice is, at least, not the worst among all possibilities. In other words, in order to maintain basic moral self-regard, people will not choose option B when option A can deliver greater benefits. There is an English-language proverb that expresses this idea of moral self-regard precisely: a bird [morally enhancing] in the hand is worth two in the bush.

The second is the diminished upside sensitivity (DUS) hypothesis. This hypothesis concerns to the rate at which outcomes of morally relevant decisions translate into moral self-regard. It is possible that the transformation of morally relevant outcomes into moral self-regard is nonlinear. Specifically, once people make a choice to avoid the worst outcome, as explained in the WOA hypothesis, they succeed in meeting the minimum moral threshold and confirm their moral self-regard. They subsequently are less motivated to keep choosing moral actions, as it is difficult to gain additional moral self-regard. This hypothesis supports the second half of the proverb: A bird in the hand is indeed more favorable than two birds in the bush. Nevertheless, with one bird in hand, people will not expend the effort to catch the second, which only brings an insignificant boost to moral self-regard.

\section{Empirical Evidence for the Two Key MTM Hypotheses in Western Individualistic Societies}

A recent study provided empirical evidence in support of the MTM. ${ }^{16}$ Using a sample from the United States; the study combined two experiments to test the above hypotheses.

The first experiment tested the WOA hypothesis. The experiment placed participants in a hypothetical investment context with random assignment to either a prosocial condition or egoistic condition. They were then asked to choose between Option A (the no-risk option) and Option B (the risky option) for themselves or the public good. The result of the experiment supports the WOA hypothesis that people refrain from choosing risky options that might bring the poor outcomes in prosocial decision-making.

The second experiment supported the DUS hypothesis. The experiment similarly re-created an investment context in requiring participants to choose whether to keep $\$ 200$ for themselves or to donate it for the public good. Similar to experiment 1, participants also needed to decide between a sure and a risky option. However, this time, there were six scenarios available to choose for the "risky" option. Each scenario provided a different possible payout ranging from $\$ 50$ to $\$ 100$, awarded in increments of $\$ 10$. These amounts were presented to every participant in ascending or descending sequence. The finding supported the DUS hypothesis. It suggested that in prosocial decision-making, people avoid making a risky decision that may generate the worst possible outcome; at the same time, people are less sensitive to the increasing benefit brought by riskier decisions compared to those made in their own interest.

\section{Is MTM Universally Applicable?}

Zlatev et $\mathrm{al}^{16}$ proposed the MTM and tested it only with participants from Western cultures (ie, the United States). We remain unsure therefore whether MTM is crossculturally applicable. It may be insufficient to help us understand moral behavioral management practices in Eastern cultures. This would limit the application breadth and domains of MTM.

For example, cross-cultural researchers have questioned whether positive self-regard is widespread outside of Western cultures. ${ }^{17}$ It may be the case that in more interdependent cultures, individual moral desire has been diluted (ie, in China's collectivist culture). Available research evidence indicates that East Asian cultures do not have the same skewed distributions of moral self-views as those in the West. ${ }^{18,19}$ Previous studies have shown that compared with individualistic cultures, people in collectivistic cultures display more holistic attention, ${ }^{20}$ a greater emphasis on social (vs personal) happiness, ${ }^{21}$ greater suspension of self-interest ${ }^{22}$ and weaker motivations toward selfexpression, self-esteem, and self-efficacy. ${ }^{17}$ These normative behavioral differences in specific cultural traits may have different social and interpersonal consequences. We 
propose that the level of interdependence in a culture may moderate the findings in the experiments above. ${ }^{1}$ Specifically, we ask, would different results appear in China's collectivist culture? The literature and research have yet to examine this question.

\section{The Current Study}

To summarize, MTM predicts that people who make moral decisions will choose an option (the sure option) that ensures a benefit with a higher value than the worst possible outcome of the available choices, instead of choosing one with a higher expected value that may produce the poorest outcome. People choose options in this way to confirm their own moral self-regard; an obtained value better than the worst possible outcome is preferable to a larger expected return not obtained. Additionally, the model predicts that once the threshold is crossed, the prosocial benefits of any other behavior will have relatively less impact on any moral decisions.

In the current study, we replicated two key experiments from the original research of Zlatev et al to determine whether the findings in the replicated MTM experiments $^{16}$ are universally applicable, or whether there will be different results in China's collectivist culture. Study 1 direct replicated the first key experiment of Zlatev et al's research. Its purpose was to examine whether people are more inclined to make decisions guaranteeing outcomes that exceed the worst possible available outcome when making prosocial (vs egoistic) decisions. Study 2 direct replicated the second key experiment of Zlatev et al and aimed to explore whether people are relatively insensitive to upside prosocial decisions. When the benefits outweigh the worst possible outcome and the beneficiary is not the self but a prosocial cause; the extent to which benefits exceed this threshold does not affect decision-making.

\section{Study I}

\section{Design and Overview of the Experiment}

Study 1 adopted a between-subject design with one factor and two levels, the Prosocial and Egoistic conditions. Participants were randomly assigned to one of the conditions to complete decision task. Study 1 compared the proportion of Option A (referring to the sure option) or Option B (referring to the risky option) in the two conditions as a preliminary test of the WOA hypothesis in in a collectivistic context.

\section{Method}

\section{Participants}

The participants were 613 college students from a local university from the southeast of China. The participants' demographic data were as follows: $58.56 \%$ were male and $41.44 \%$ were female; the mean age of the sample was 20.77 years with a standard deviation of 1.51. Twentynine participants had to be excluded as their response times were sample outliers. The participants took part in an online experiment via a reliable Chinese data-collection platform similar to Qualtrics Online Sample; the platform's URL is www.credamo.com. Each participant provided written informed consent to take part in the experiment. The Ethics Committee of the College of Teacher Education, Zhejiang Normal University approved the study.

\section{Procedure}

Participants began the experiment by reading descriptions of different decision situations. In the Prosocial condition, the participants read: Imagine that you work for a charity that is in clear need of support. The charity receives a gift of 2000 yuan and you are deciding what to do with this money. In the Egoistic condition, the participants read: Imagine that you receive a gift of 2000 yuan and that you are deciding what to do with this money. After reading the different decision situations, the participants were given the same two options. They also learned that they were deciding between a no-risk option and a risky option.

If the participant selected the no-risk option, then the money would be put into the charity's (Prosocial Condition) or their own (Egoistic condition) savings account, in which case they would be able to ensure that they could keep 2000 yuan or give it to the charity. If the risky option was selected, then the money would be invested. In this case, there was a $75 \%$ chance that the charity or the individual would receive 2750 yuan and a $25 \%$ chance they would receive 0 yuan. Compared with the no-risk option (2000 yuan), the risky option had a higher expected value (2062.5 yuan). The risky option, however, might also lead to the worst possible outcome.

A seven-point scale was then administered that asked participants to report which option they preferred between Option A and Option B in both conditions: 1 signified "Prefer Option A" and 7 signified "Prefer Option B." The materials were those created by Zlatev's team. The first author obtained permission from Zlatev to replicate and use their experiment materials in the current study. 
The appropriate attribution was made to the creators of the materials.

\section{Statistical Analysis Strategy}

Statistical analysis was performed using the $\mathrm{R}$ programming language. The analysis code was obtained from osf.io/38zy2/, which was provided by Zlatev's team. Binomial logistic regression was used examine the proportion of those choosing the no-risk versus the risky option. An independent sample $t$-test was conducted to investigate the continuous measure. The effect sizes for binomial logistic regression (Cramer's v) and t-tests (Cohen's $d$ ) were calculated.

\section{Results}

The result of binomial logistic regression analysis showed that, compared with the prosocial condition (20.07\%), participants were more likely to choose the risky option $(30.00 \%)$ in the egoistic condition, $X^{2}[d f=1, \mathrm{~N}=584]=$ $7.12, p=0.008$, Cramer's $v=0.11$.

The results of the independent sample $t$-test further corroborated the finding, showing that participants were more preferred to choose the no-risk option in the prosocial condition than the participants in the egoistic condition, $t$ [582] $=-2.10, p=0.036$, Cohen's $d=-0.17, M$ prosocial $=3.36, S E_{\text {prosocial }}=0.10, M_{\text {egoistic }}=3.06, S E$ egoistic $=0.10$.

\section{Discussion}

Study 1 supports the first hypothesis of the MTM. The hypothesis proposes the outcome of the decision must confirm the individual's moral self-regard and so must be better than the worst possible outcome. In general, individuals were more hesitant to make decisions that were likely to result in the worst possible outcome when the choice had ethical applications and did not involve pure self-interest. This was true even though the decision forfeited the larger expected value, which was objectively better.

\section{Study 2}

\section{Design and Overview of the Experiment}

The DUS hypothesis was tested in Study 2, which holds that when individuals make prosocial (vs purely egoistic) choices, they are relatively insensitive to the possible beneficial effects of the larger advantage. We propose that these results might be influenced by Eastern culture.

\section{Method}

\section{Participants}

One thousand one hundred and forty-eight college students from a university in the southeast of China took part in the experiment via Credamo. The participants' demographics were as follows: $62.73 \%$ male and $37.49 \%$ female; the mean age of the sample was 20.61 years with a standard deviation of 1.39. Six participants were excluded as they did not complete the demographic questions. Similarly, 51 participants were excluded because their response times were sample outliers. Each participant provided written informed consent to take part in the current experiment. The Ethics Committee of the College of Teacher Education, Zhejiang Normal University approved the current study.

\section{Procedure}

Similar to Study 1, participants were randomly assigned to either an Egoistic or Prosocial condition and directed to decide how to manage money between Options A or B. The Study 1 descriptions of different decision situations were provided to them. In Study 2, however, the participants had to decide for all six separate scenarios. The difference was that the risky option was associated with higher monetary value as compared to study 1 . This amount was between 2500 yuan and 3000 yuan and varied in increments of 100 yuan. The amounts were displayed to each participant in increasing or decreasing order during the scenarios (see Figure 1). As was the case in Study 1, appropriate attributions were made to Zlatev's team and the first author obtained the necessary permissions to replicate and use their experimental materials in this study.

\section{Statistical Analysis}

Statistical analysis was performed using the $\mathrm{R}$ programming language. The analysis code was obtained from osf.io/38zy2/, which was provided by Zlatev's team. A mixed-effects binary logistic regression was used to examine the DUS hypothesis. Simple slopes analysis was conducted to evaluate whether the upside amount of the risky option affected the decisions.

\section{Results}

A binary logistic regression, which set the participants as a random effect, revealed a main effect that participants were more likely to take the risky option as the level of risk increased $(b=0.57, z=14.89, p<0.001)$. It did not, however, show significant interaction effects in accordance 


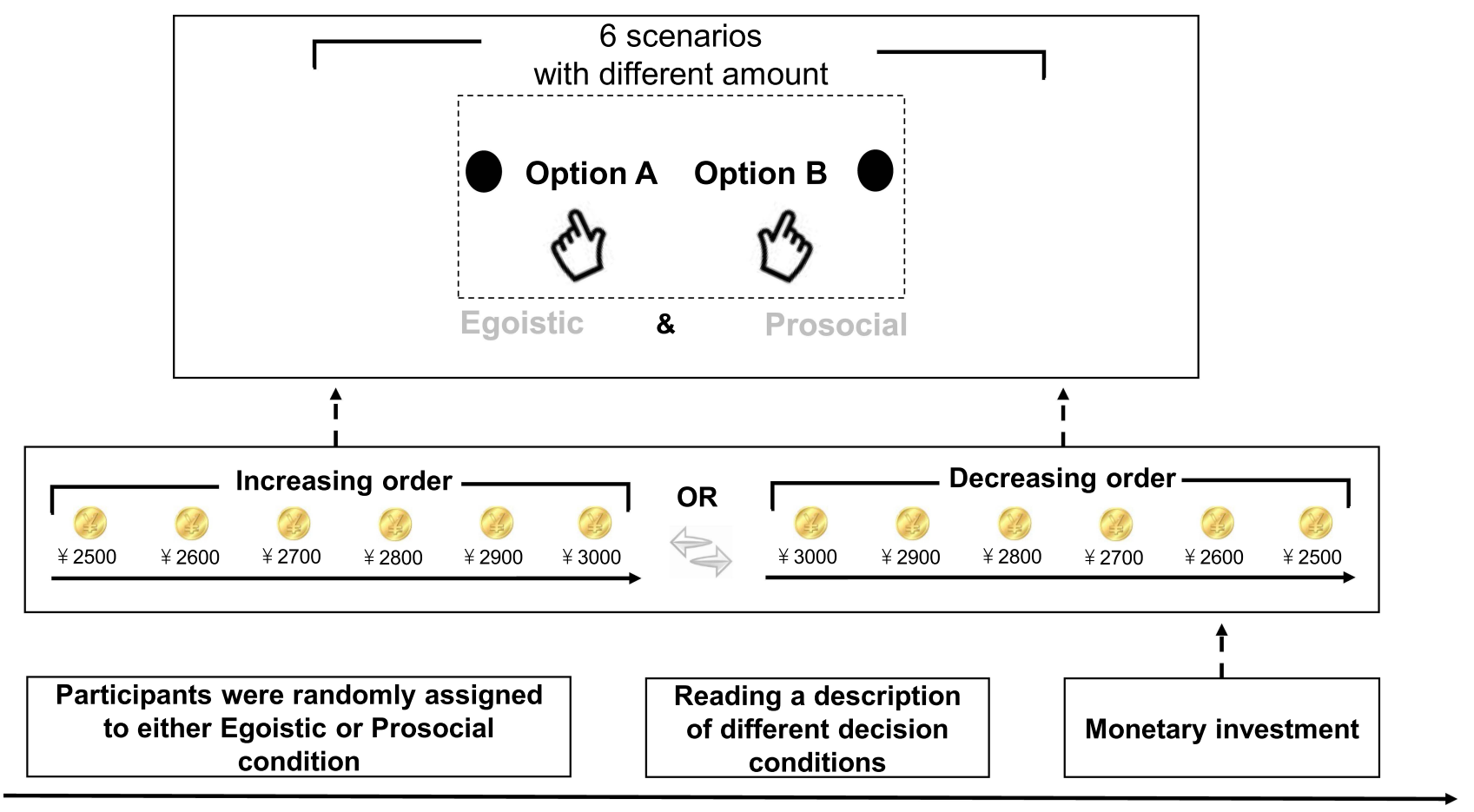

Figure I Procedure and experimental task of study 2. A monetary investment task was applied in the test context.

with the DUS hypothesis $(b=-0.06, z=0.83, p=0.409)$. Simple slopes analyses indicated that participants' decisions in the egoistic condition $(b=0.60, z=11.53, p<$ 0.001 ) were more likely to be influenced by the upside amount of the risky option than they were in the prosocial condition $(b=0.54, z=9.76, p<0.001$; more details can be seen in Figure 2).

\section{Discussion}

Study 2 partly supports the MTM's second hypothesis. We did not detect a significant interaction, though the beta coefficient for the egoistic condition was slightly larger than that for the prosocial condition. The DUS hypothesis suggested that, when individuals' moral selfrespect is undermined, the decisions of the members are less sensitive to the available benefits they may receive. These benefits are more compelling than the least rewarding advantage in the decision set. It should be noted nonetheless that individuals in the prosocial condition were not completely indifferent to the potential benefits of risky choices: They were inclined to select risky alternatives that have substantial potential gains rather than ones with lower value but guaranteed potential gains. However, compared within the egoistic condition, this impact was essentially modest.

\section{General Discussion}

In the current study, two experiments were conducted to examine the question "Is the MTM universally applicable?" Specifically, we focused on whether there are different patterns of decisions and results among members of China's collectivist culture. Study 1 supported the MTM's first hypothesis. The results showed that when the beneficiary was a prosocial cause, people preferred the smaller guaranteed positive outcomes to the larger uncertain ones. Study 2 partly supported the DUS hypothesis of the MTM. The results revealed that people were sensitive to the maximum potential benefits, to a degree, when making decisions exclusively for themselves as opposed making them for a charity.

\section{The WOA Hypothesis of MTM Can Be Applied in Western and Eastern Cultures} Ours is the first replicated experiment that tested the WOA hypothesis of MTM with a Chinese sample. The results showed consistency with those of Western research. ${ }^{16}$ In the current study, if the recipient was a prosocial cause; the participants preferred the option offering the (no-risk) guaranteed positive results rather than the risky one. The WOA hypothesis of MTM proposes that individuals avoid making decisions that are 


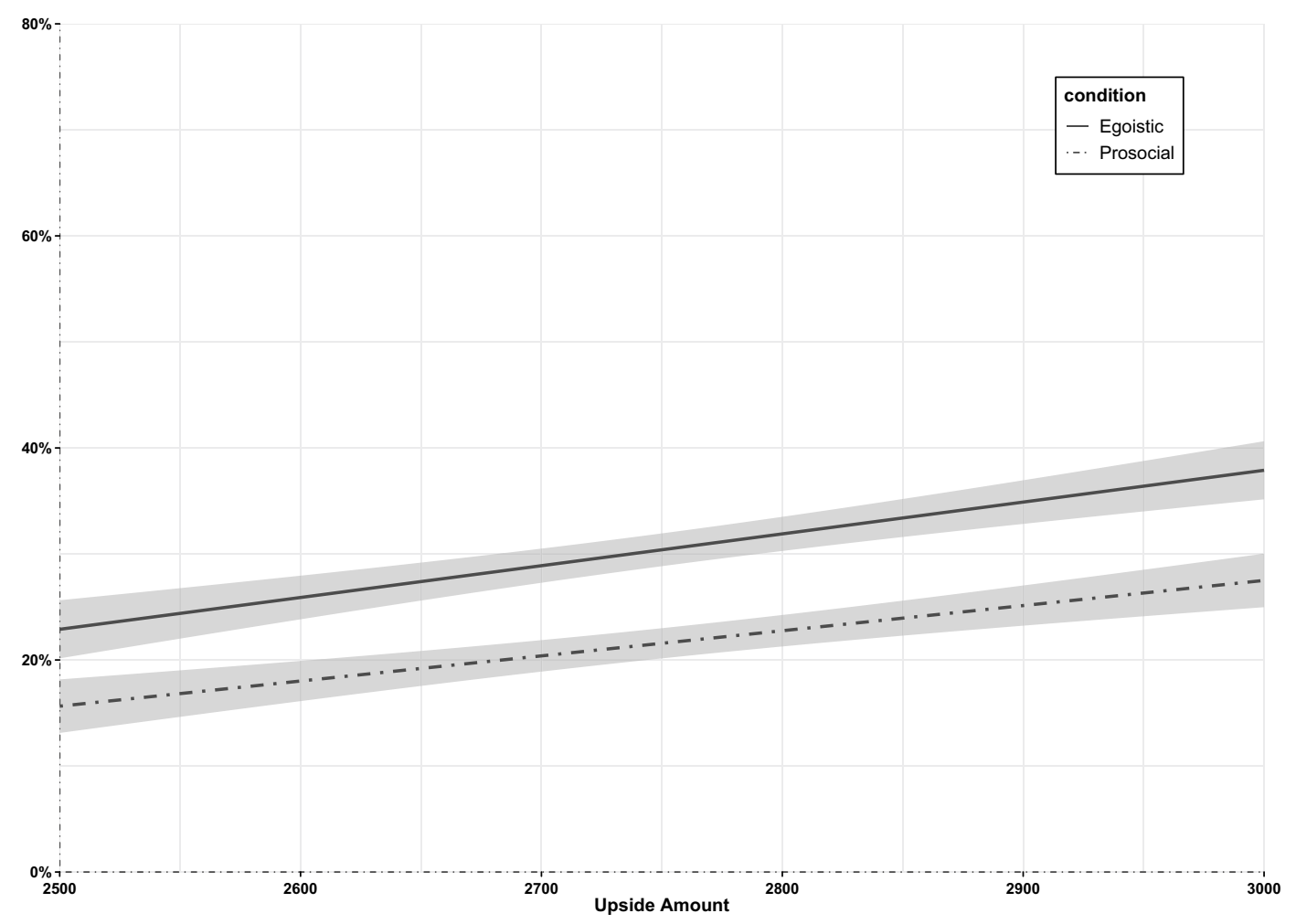

Figure 2 Percentage of participants choosing the risky option. Percentage of participants in study 2 choosing the risky option as a function of the condition and the risky option's upside amount. The solid line indicates the egoistic (self) condition and the dot dash line indicates the prosocial (charity) condition. The unit of money is yuan.

likely to bring about the worst possible result to the potential beneficiary. As is the case with other social psychological models, the MTM is not intended to cover every conceivable aspect of a situation that might be considered when making a decision. The analyses predict that once individuals' ethical self-respect is undermined and their commitment reaches the level of the underlying limit indicated by the WOA hypothesis, their choices are less influenced by potential benefit.

Why do individuals make such decisions? What is the universal motivation behind the behavior pattern? Moral self-regard refers to the degree to which individuals believe that they have positive moral qualities. ${ }^{1}$ Research evidence has shown that East Asians' moral self-views differ from those common to members of Western culture. ${ }^{18,19}$ In the current experiment, we did not find evidence for the claim that moral self-regard is a universal motivation of individuals making risky decisions in moral vs non-moral domains. It remains unknown whether moral self-regard is a universal motivation for moral behavior outside of Western cultures. ${ }^{17}$ Future research should continue to examine whether moral self-regard is a universal, non-culture specific motivation.

\section{The Normative Cultural Differences and the Amounts of Money Used May Have Caused the Non-Significant Interaction for the DUS Hypothesis}

The DUS hypothesis of MTM proposes that individuals may shy away from the choice of a much bigger potential gain, to avoid the worst possible outcome in order to preserve their moral self-regard but will start making risky choices once they have met minimum threshold. The second experiment evaluated the DUS hypothesis of MTM under China's collectivist culture. The results suggested that when individuals decide on the choices for themselves, the choices were more sensitive to the maximum potential benefit than when making the decisions for a charity or another prosocial recipient. The results were partially inconsistent with the original research. ${ }^{16}$ Specifically, even though the beta coefficient for the egoistic condition was slightly larger than the one for the prosocial condition, we did not detect a significant interaction in study 2 .

One potential reason for the lack of a significant interaction may have been normative cultural differences in culture-specific behavioral traits. Previous studies have 
indicated that European Americans place a strong normative emphasis on personal independence, while Asians put much greater emphasis on interdependence of the self with others. ${ }^{23}$ Many previous studies have shown that compared with individualistic cultures, people in collectivistic cultural contexts place greater emphasis on social (vs personal) happiness ${ }^{21}$ and engage in greater suspension of self-interest. $^{22}$ These normative cultural differences in culture-specific behavioral traits may explain why no significant interaction effects were observed between the conditions. Future research should investigate the potential effects of the suspension of self-interest and emphasis on social (vs personal) happiness of individuals in individualistic cultures.

Another possible reason for the lack of a significant interaction may been the amounts of money used. Study 2 used increments of 100 yuan (approximately 15.50 USD), which was about 1.5 times larger than the increments in the original research (10 USD). Moreover, the range from the lowest amount (2500 yuan) to the highest amount (3000 yuan) was about $\$ 77.50$ USD in our study (compared to \$50 USD in the original study), which also was 1.5 times larger than the original range. It should also be noted that the amounts in the no-risk option (2000 yuan) and the risk option (about 3000 yuan) were almost $35 \%$ and $51 \%$ of the gross national product per month, respectively, in China according to 2019 statistics. In the original research, the amounts of money used were only $5 \%$ or $7 \%$ of the gross national product per month according to 2019 statistics of the United States. These differences may account for the lack of a significant interaction in Study 2. The potential impact of the amounts of money used in the current study should be further explored.

\section{Chinese Results Pattern Unaffected by Uncertainty Effects or Risk Aversion}

Many prosocial choices require a person to hand over their welfare to another willfully. The act of offering one's welfare to another, however, can be seen as a loss of control, thus increasing uncertainty. ${ }^{24}$ People's risk preferences in decision-making for others, based on clearly described odds and outcomes, are often different from their own. ${ }^{25}$ Whether such factors (ie, uncertainty effects, risk aversion) influenced the results of the current study is a question that remains unanswered.
To some degree, the influence of these factors was excluded from the risk context in which our predictions were tested. As mentioned in the two experiments' methods (the money is gifted), equal costs of all prosocial behaviors decouple the value of individuals' moral selfregard from the prosocial benefit generated by the personal cost. This occurs when people face a choice between an uncertain but greater contribution to a specific prosocial cause versus providing a certain but small donation to it. Although the material cost of doing good in the two experiments is the same, the psychological costs incurred by choosing the larger and riskier donation may lead people to choose to the smallest possible donation.

Second, the comparison between prosocial risk and purely egoistic decision-making enables us to evaluate whether the prosocial risk aversion patterns predicted by the MTM are identical to the well-known risk aversion and uncertainty effects for decisions that only affect the self. ${ }^{24-27}$ Moreover, the probabilities and expected values of prosocial and egoistic choices with the same structure reduce the likelihood that an individual's particular qualities in the risky tradeoffs lead to potentially harmful decisions. We have therefore reduced the impact of these variables in the current study to some degree. This means that the pattern of the results observed in the current study was not affected by uncertain effects, risk aversion, or the specific values in risk tradeoffs.

\section{Limitations and Future Directions}

In summary, this study examines the MTM in the context of China's collectivist culture and provides a deeper understanding of this model. It is important to note a few limitations. First, in the experimental task, the participants in the egoistic condition (choice between giving to the charity or themselves) had a $75 \%$ chance of receiving a certain amount of money and a $25 \%$ chance of receiving nothing. Whether different ratios of loss to gain (ie, $80 \%$ vs $20 \%$ ) influence the participant's decision-making is worth exploring in future studies. Second, the two main hypotheses of the MTM were tested in a hypothetical investment context in the current study. Whether there would be differing results in a real money environment should be investigated in the future. Moreover, in the current study, we did not obtain enough evidence to support the claim that moral self-regard is a universal motivation for the individuals making risky decisions in moral vs non-moral domains. It is important to explore the universal motivation behind the behavior pattern. Finally, while our participants came from China, 
we recruited only college students for the study's decision task. As data were only collected from university students, the applicability of the results should be evaluated with more representative samples in the future.

\section{Conclusion}

In the current study, two direct replication experiments were conducted to examine the question "Is the MTM universally applicable?" Specifically, we focused on whether there are different patterns of decision results found in China's collectivist culture. Study 1 supported the MTM's WOA hypothesis, while study 2 partly supported the DUS hypothesis of the MTM. The current study provides initial evidence for the validity of using the worst outcome avoidance (WOA) hypothesis of MTM among China's collectivist culture, indicating that the WOA hypothesis MTM may be applied universally.

\section{Ethics Statement and Informed Consent}

This study was conducted in accordance with the Declaration of Helsinki. The Ethics Committee of College of Teacher Education, Zhejiang Normal University approved the study. The participants interested in participating read the informed consent which contained the objectives, benefits, and risks of participation; those who agreed to participate in the study gave their informed consent, accepting to continue with the survey. The participants were informed that their responses to task would be anonymous and confidential, and the data collected would be used for academic research only.

\section{Acknowledgments}

We thank the participants of this study for their time and contributions. Moreover, we thank Zlatev and his team allow us to use relevant materials and analysis codes to carry out this research. In addition, we thank Ms. Yu Wang for her help in reviewing and Ms. Xue Lin for her help in data collection.

\section{Author Contributions}

Weilong Xiao and Binghai Sun should be considered cofirst authors. All authors made substantial contributions to conception and design, acquisition of data, or analysis and interpretation of data; took part in drafting the article or revising it critically for important intellectual content; agreed to submit to the current journal; gave final approval of the version to be published; and agree to be accountable for all aspects of the work.

\section{Funding}

This work was supported by Major Support Projects for Emerging (Interdisciplinary) Disciplines of Philosophy and Social Sciences of Zhejiang Province in China [Grant numbers: 19XXJC04ZD-3].

\section{Disclosure}

Authors report no conflicts of interest in this work.

\section{References}

1. Monin B, Jordan AH. Dynamic moral identity: a social psychological perspective. In: Narvaez D, Lapsley D, editors. Personality, Identity, and Character: Exploration in Moral Psychology. Cambridge, UK: University Press; 2009:341-354.

2. Mazar N, Amir O, Ariely D. The dishonesty of honest people: a theory of self-concept maintenance. $J$ Mark Res. 2008;45:633-644. doi:10.1509/jmkr.45.6.633

3. Aquino K, Reed A II. The self-importance of moral identity. $J$ Pers Soc Psychol. 2002;83:1423-1440. doi:10.1037/0022-3514.83.6.1423

4. Aquino K, McFerran B, Laven M. Moral identity and the experience of moral elevation in response to acts of uncommon goodness. J Pers Soc Psychol. 2011;100:703-718. doi:10.1037/a0022540

5. Jordan J, Mullen E, Murnighan JK. Striving for the moral self: the effects of recalling past moral actions on future moral behavior. Pers Soc Psychol Bull. 2011;37:701-713. doi:10.1177/0146167211400208

6. Zhong CB, Liljenquist K. Washing away your sins: threatened morality and physical cleansing. Science. 2006;313:1451-1452. doi:10.1126/science. 1130726

7. Monin B, Sawyer PJ, Marquez MJ. The rejection of moral rebels: resenting those who do the right thing. J Pers Soc Psychol. 2008;95:76-93. doi:10.1037/0022-3514.95.1.76

8. Miller DT, Monin B. Moral opportunities versus moral tests. In: van Lange P, Forgas J, Jussim L, editors. The Social Psychology of Morality. New York, NY: Psychology Press; 2016:40-55.

9. Lin SC, Zlatev JJ, Miller DT. Moral traps: when self-serving attributions backfire in prosocial behavior. $J$ Exp Soc Psychol. 2017;70:198-203. doi:10.1016/j.jesp.2016.11.004

10. Shalvi S, Gino F, Barkan R, Ayal S. Self-serving justifications: doing wrong and feeling moral. Curr Dir Psychol Sci. 2015;24:125-130. doi: $10.1177 / 0963721414553264$

11. Ploner M, Regner T. Self-image and moral balancing: an experimental analysis. J Econ Behav Organ. 2013;93:374-383. doi:10.1016/j. jebo.2013.03.030

12. Shalvi S, Dana J, Handgraaf MJ, De Dreu CK. Justified ethicality: observing desired counterfactuals modifies ethical perceptions and behavior. Organ Behav Hum Decis Process. 2011;115:181-190. doi:10.1016/j.obhdp.2011.02.001

13. Alleyne E, Fernandes I, Pritchard E. Denying humanness to victims: how gang members justify violent behavior. Group Process Intergroup Relat. 2014;17:750-762. doi:10.1177/1368430214536064

14. Tsang JA. Moral rationalization and the integration of situational factors and psychological processes in immoral behavior. Rev Gen Psychol. 2002;6:25-50. doi:10.1037/1089-2680.6.1.25

15. Sachdeva S, Iliev R, Medin DL. Sinning saints and saintly sinners: the paradox of moral self-regulation. Psychol Sci. 2009;20:523-528. doi:10.1111/j.1467-9280.2009.02326.x 
16. Zlatev JJ, Kupor DM, Laurin K, Miller DT. Being "good" or "good enough": prosocial risk and the structure of moral self-regard. $J$ Pers Soc Psychol. 2020;118:242-253. doi:10.1037/pspa0000175

17. Heine SJ, Lehman DR, Markus HR, Kitayama S. Is the rea universal need for positive self-regard? Psychol Rev. 1999;106:766-794. doi:10.1037/0033-295X.106.4.766

18. Campbell JD, Trapnell PD, Heine SJ, Katz IM, Lavallee LF, Lehman DR. Self-concept clarity: measurement, personality correlates, and cultural boundaries. J Pers Soc Psychol. 1996;70:141-156. doi: $10.1037 / 0022-3514.70 .1 .141$

19. Diener E, Diener M. Cross-cultural correlates of life satisfaction and self-esteem. In: Culture and Well-Being. Dordrecht: Springer; 2009:71-91.

20. Masuda T, Nisbett RE. Attending holistically versus analytically: comparing the context sensitivity of Japanese and Americans. $J$ Pers Soc Psychol. 2001;81(5):922-934. doi:10.1037/00223514.81.5.922

21. Kitayama S, Park H, Sevincer AT, Karasawa M, Uskul AK. A cultural task analysis of implicit independence: comparing North America, Western Europe, and East Asia. J Pers Soc Psychol. 2009;97(2):236-255. doi:10.1037/a0015999
22. Kitayama S, Park J. Error-related brain activity reveals self-centric motivation: culture matters. J Exp Psychol Gen. 2014;143(1):62-70. doi:10.1037/a0031696

23. Kitayama S, King A, Yoon C, Tompson S, Huff S, Liberzon I. The dopamine D4 receptor gene (DRD4) moderates cultural difference in independent versus interdependent social orientation. Psychol Sci. 2014;25(6):1169-1177. doi:10.1177/0956797614528338

24. Vives ML, FeldmanHall O. Tolerance to ambiguous uncertainty predicts prosocial behavior. Nat Commun. 2018;9:1-9. doi:10.1038/ s41467-018-04631-9

25. Olschewski S, Dietsch M, Ludvig EA. Anti-social motives explain increased risk aversion for others in decisions from experience. Judgm Decis Mak. 2019;14:58-71.

26. Rabin M. Risk aversion and expected utility: a calibration theorem. Econometrica. 2000;68:1281-1292. doi:10.1111/1468-0262.00158

27. Tversky A, Kahneman D. Rational choice and the framing of decisions. J Bus. 1986;59:251-278. doi:10.1086/296365
Psychology Research and Behavior Management

\section{Publish your work in this journal}

Psychology Research and Behavior Management is an international, peer-reviewed, open access journal focusing on the science of psychology and its application in behavior management to develop improved outcomes in the clinical, educational, sports and business arenas. Specific topics covered in the journal include: Neuroscience, memory and decision making; Behavior modification and management; Clinical

\section{Dovepress}

applications; Business and sports performance management; Social and developmental studies; Animal studies. The manuscript management system is completely online and includes a very quick and fair peer-review system, which is all easy to use. Visit http://www. dovepress.com/testimonials.php to read real quotes from published authors. 